medical-waste management services in the United States. The company's proprietary electro-thermal-deactivation process destroys human pathogens without producing harmful airborne emissions and permits resource recovery. Stericycle operates on a multiregional basis, providing medical-waste collection, transportation, treatment, disposal, reduction, and resource recovery.

FROM: Washington State Department of Health. Press Release: Probable transmission of occupationally acquired tuberculosis at Stericyle Inc, a medical waste processing facility in Morton, WA. Olympia, WA: Washington State Department of Health. March 4, 1998.

\section{Vancomycin-Resistant Enterococci From Community Sources}

Researchers from the Erasmus University Medical Center in Rotterdam, The Netherlands, reported the results of a study to determine the prevalence of vancomycin-resistant enterococci (VRE) in The Netherlands. Six hundred twenty-four hospitalized patients from intensive-care units or hemato-oncology wards in nine hospitals and 200 patients living in the community were screened for VRE colonization.

Enterococci were found in $49 \%$ of the hospitalized patients and in $80 \%$ of the patients living in the community. Of these strains, $43 \%$ and $32 \%$, respectively, were Enterococcus faecium. VRE were isolated from 12 (2\%) of 624 and 4 (2\%) of 200 hospitalized patients and patients living in the community, respectively. Polymerase chain reaction (PCR) analysis of these 16 strains and 11 additional clinical VRE isolates from one of the participating hospitals revealed 24 van $\mathrm{A}$ gene-containing, 1 van $\mathrm{B}$ gene-containing, and 2 van $\mathrm{C} 1$ gene-containing strains. All strains were cross-resistant to avoparcin but were sensitive to the novel glycopeptide antibiotic LY333328. Genotyping of the strains by arbitrarily primed PCR and pulsed-field gel electrophoresis revealed a high degree of genetic heterogeneity. These findings underscore a lack of hospital-driven endemicity of VRE clones.

The authors believe that this data suggests that the VRE in hospitalized patients have originated from unknown sources in the community.

FROM: Endtz HP, van den Braak N, van Belkum A, Kluytmans JA, Koeleman JG, Spanjaard L, et al. Fecal carriage of vancomycin-resistant enterococci in hospitalized patients and those living in the community in The Netherlands. J Clin Microbiol 1997;35:3026-3031.

\section{Influenza Virus A (H5N1) Risk Factors}

The Department of Health in Hong Kong and the Centers for Disease Control and Prevention in Atlanta, Georgia, reported the results of a case-control study to determine risk factors associated with recent cases of avian influenza in Hong Kong.

The case-control study was aimed at comparing different exposure risk factors between patients and controls. It covered a number of areas including live-poultry exposure, exposure when preparing food, food eaten during the week before onset, and human-illness exposure during the week before onset. The results indicated that visiting a poultry stall in the week before becoming ill was the strongest risk factor. These results support earlier findings that human-tohuman transmission of the virus is inefficient.

A total of 18 cases of influenza A(H5N1) was reported in Hong Kong. The day of onset of illness of the last case was December 28, 1998. A 24-year-old female patient is still under treatment and in stable condition, while 11 others have been discharged after recovery. Six people died of the disease.

FROM: Chin J (World Health Organization). Influenza virus A (H5N1) risk factors. ProMED-e-mail post; March 12, 1998.

\section{Hospital Epidemic of Malassezia pachydermatis}

Investigators from CDC's Hospital Infections Program recently reported an unusual outbreak involving the yeast Malassezia pachydermatis. Malassezia species are lipophilic yeasts that are emerging as nosocomial pathogens, particularly in low-birth-weight neonates who receive lipid emulsions.

A cluster of patients with $M$ pachydermatis infection was identified in an intensive-care nursery, and an investigation was initiated. A case patient was defined as any infant in the intensive-care nursery who had a positive culture for $M$ pachydermatis between October 17, 1993, and January 18, 1995. A cohort study was conducted to identify risk factors for colonization and infection with $M$ pachydermatis. Cultures were collected from the infants and the healthcare workers and from the healthcare workers' pets, because this organism has been associated with otitis external in dogs.

Fifteen infants met the case definition: eight with bloodstream infections, two with urinary tract infections, one with meningitis, and four with asymptomatic colonization. The case patients were significantly more likely than the other infants to weigh $1,300 \mathrm{~g}$ or less $(15 / 65$ vs $0 / 419$; $P<.001$ ). In a multivariate analysis of infants weighing 1,300 $\mathrm{g}$ or less, the independent risk factors for colonization or infection with $M$ pachydermatis were a greater severity of concomitant illness, arterial catheterization for 9 or more days, and exposure to nurse A.

In a point-prevalence survey, 9 additional infants, 1 healthcare worker, and 12 of the healthcare workers' pet dogs had positive cultures for $M$ pachydermatis. The isolates from all 15 case patients, the 9 additional colonized infants, 1 healthcare worker, and 3 of the 12 dogs had identical patterns of restriction fragment-length polymorphisms.

It was concluded that it is likely that $M$ pachydermatis was introduced into the intensive-care nursery on healthcare workers' hands after being colonized from pet dogs at home. The organism persisted in the nursery through patient-to-patient transmission.

FROM: Chang HJ, Miller HL, Watkins N, Arduino MJ, Ashford DA, Midgley G, et al. An epidemic of Malassezia 